\title{
Graph Classes Characterized Both by Forbidden Subgraphs and Degree Sequences
}

\author{
Michael D. Barrus, ${ }^{1}$ Mohit Kumbhat, ${ }^{1}$ and Stephen G. Hartke ${ }^{2}$ \\ ${ }^{1}$ DEPARTMENT OF MATHEMATICS \\ UNIVERSITY OF ILLINOIS \\ URBANA, ILLINOIS 61801 \\ E-mail:mbarrus2@math.uiuc.edu; kumbhat2@math.uiuc.edu \\ 2 DEPARTMENT OF MATHEMATICS \\ UNIVERSITY OF NEBRASKA \\ LINCOLN, NEBRASKA 68588 \\ E-mail: hartke@unl.edu
}

Received November 23, 2005; Revised July 28, 2007

Published online 15 November 2007 in Wiley InterScience(www.interscience.wiley.com).

DOI 10.1002/jgt.20270

Abstract: Given a set $\mathcal{F}$ of graphs, a graph $G$ is $\mathcal{F}$-free if $G$ does not
contain any member of $\mathcal{F}$ as an induced subgraph. We say that $\mathcal{F}$ is a
degree-sequence-forcing set if, for each graph $G$ in the class $\mathcal{C}$ of $\mathcal{F}$-free
graphs, every realization of the degree sequence of $G$ is also in $\mathcal{C}$. We give
a complete characterization of the degree-sequence-forcing sets $\mathcal{F}$ when
$\mathcal{F}$ has cardinality at most two. $\odot 2007$ Wiley Periodicals, Inc. J Graph Theory $57: 131-148,2008$ Keywords: degree-sequence-forcing set; forbidden subgraphs; degree sequence characterization; 2switch; potentially P-graphic; forcibly P-graphic

Contract grant sponsor: National Science Foundation VIGRE (to M.D.B.); Contract grant number: DMS 9983160.

Journal of Graph Theory

(C) 2007 Wiley Periodicals, Inc. 


\section{INTRODUCTION}

Given a collection $\mathcal{F}$ of graphs, a graph $G$ is said to be $\mathcal{F}$-free if $G$ contains no induced subgraph isomorphic to an element of $\mathcal{F}$. We say that elements of $\mathcal{F}$ are forbidden subgraphs for the class of $\mathcal{F}$-free graphs. Forbidden subgraph notions have proven fruitful in graph theory; Kuratowski's Theorem can be rephrased as a statement of which induced subgraphs are forbidden for planar graphs, and the Strong Perfect Graph Theorem [2] characterizes perfect graphs in terms of their forbidden subgraphs.

The degree sequence $d(G)$ of a graph $G$ is the list $\left(d_{1}, d_{2}, \ldots, d_{n}\right)$ of degrees of the vertices of $G$, written in nonincreasing order. We say a graph class $\mathcal{C}$ has a degree sequence characterization if it is possible to determine whether or not a graph $G$ is in $\mathcal{C}$ based solely on the degree sequence of $G$. Degree sequence characterizations do not exist for most graph classes, but are extremely useful when they do exist.

In this article, we address the question of which classes of graphs can be characterized both in terms of a small collection of forbidden subgraphs, and in terms of their degree sequences. More precisely, let us define a collection $\mathcal{F}$ of graphs to be degree-sequence-forcing if whenever any realization of a graphic sequence $\pi$ is $\mathcal{F}$-free, every other realization of $\pi$ is $\mathcal{F}$-free as well. We characterize the degree-sequence-forcing sets of order at most two.

One well-known degree-sequence-forcing set that motivated our interest in this question is $\left\{2 K_{2}, C_{4}, C_{5}\right\}$. The class of $\left\{2 K_{2}, C_{4}, C_{5}\right\}$-free graphs has been shown by Földes and Hammer [7] to be exactly the class of split graphs: the graphs whose vertex sets can be partitioned into a clique and an independent set. Many results are known about the split graphs. Of particular interest is the characterization by Hammer and Simeone [11] of split graphs entirely in terms of their degree sequences: if $G$ is a graph having degree sequence $d(G)=\left(d_{1}, \ldots, d_{n}\right)$ in nonincreasing order, then $G$ is split if and only if

$$
\sum_{i=1}^{m} d_{i}=m(m-1)+\sum_{i=m+1}^{n} d_{i},
$$

where $m=\max \left\{k: d_{k} \geq k-1\right\}$.

This property of having two characterizations-one in terms of forbidden subgraphs, the other in terms of degree sequences-is not unique to the class of split graphs. A threshold sequence is a graphic sequence which is not majorized by any other graphic sequence, and a graph $G$ is said to be a threshold graph if $d(G)$ is a threshold sequence. It is not surprising, perhaps, that the threshold graphs can be characterized in terms of their degree sequences; such a characterization was given by Hammer et al. [10]. They can be characterized, moreover, by their forbidden subgraphs; Chvátal and Hammer [4] showed a graph is threshold if and only if it is $\left\{2 K_{2}, C_{4}, P_{4}\right\}$-free.

A superclass of both the split and the threshold graphs is the class of pseudosplit graphs. Maffray and Preissman [15] defined the pseudo-split graphs to be the 
$\left\{2 K_{2}, C_{4}\right\}$-free graphs and showed they have a degree sequence characterization similar to those of the split graphs and threshold graphs.

One reason for the interest in finding degree sequence characterizations of graph classes is for quick recognition algorithms. Testing whether a graph is $\mathcal{F}$-free takes polynomial time, but the degree is dependent on the order of the largest element of $\mathcal{F}$, assuming $\mathcal{F}$ is finite (McKee [16] gives a necessary and sufficient condition for characterizing a graph class in terms of finitely many forbidden subgraphs). In contrast, degree sequence characterizations usually give rise to linear time recognition algorithms, as in the case of the split, threshold, and pseudo-split graphs.

In Theorems 22 and 24, we give a complete characterization of the degreesequence-forcing sets $\mathcal{F}$ when $\mathcal{F}$ has cardinality at most two. Our results are particularly interesting in light of recent work by others determining which sets $\mathcal{F}$ of graphs force the class of $\mathcal{F}$-free graphs to have certain properties. Faudree et al. [5,6] characterized the sets $\mathcal{F}$ of graphs where the class of $\mathcal{F}$-free graphs are Hamiltonian, and Randerath [17] characterized pairs $\{A, B\}$ such that $\{A, B\}$ free graphs are 3-colorable. Moreover, Gould et al. [9] characterized all pairs of connected graphs $\{A, B\}$ such that any 3 -connected $\{A, B\}$-free graph is pancyclic.

The concept of degree-sequence-forcing sets can also be stated in the language of degree sequence problems. Given a graph-theoretic property $P$ invariant under isomorphism, a graphic sequence $\pi$ is said to be potentially $P$-graphic if there exists a realization of $\pi$ having property $P$, and forcibly $P$-graphic if every realization of $\pi$ has property $P$. Potentially and/or forcibly $P$-graphic sequences have been characterized for many properties $P$, such as planarity [22], $k$-connectedness, and Hamiltonicity. In [3] and [20], Chernyak, Chernyak, and Tyshkevich characterize forcibly $P$-graphic sequences for the properties $P$ of chordality, strong chordality, intervality, comparability, and trivial perfectness. Zverovich [23] and Zverovich and Urbanovich [24] have also classified forcibly 3-colorable sequences and forcibly 2-matroidal sequences. The survey of Rao [18] presents additional results on potentially and forcibly $P$-graphic sequences and provides an extensive bibliography.

Forcibly $P$-graphic sequences are clearly potentially $P$-graphic, though the converse in general is not true; consider, for example, when $P$ is the property of planarity. Our goal in this article is to characterize the classes $\mathcal{F}$ such that potentially $\mathcal{F}$-free sequences are forcibly $\mathcal{F}$-free.

\section{PRELIMINARIES}

In this article, we follow the terminology and notation of West [21]. In particular, for any graph $G$ and a vertex $v$ of $G$, we use $n(G), d_{G}(v)$, and $d(G)=\left(d_{1}, d_{2}, \ldots, d_{n}\right)$ to denote the number of vertices in $G$, the degree of $v$ in $G$, and the degree sequence of $G$ in non-increasing order, respectively. We shall denote the disjoint union of two graphs $G$ and $H$ by $G+H$. We consider all graphs to be simple and to have at least one vertex, particularly when considering induced subgraphs. We will often 
use "graph" to mean "isomorphism class of graphs," rather than a specific element of a given isomorphism class. We trust that the context will make it clear which meaning is intended.

We say a graph $G$ is $A$-free if there is no induced copy of $A$ in $G$ and $\{A, B\}$-free if there is no induced copy of $A$ or of $B$ in $G$. We say that a set $\mathcal{F}$ of graphs is degreesequence-forcing if whenever a graphic sequence $\pi$ has an $\mathcal{F}$-free realization, then every realization of $\pi$ is $\mathcal{F}$-free. Equivalently, a set $\mathcal{F}$ is degree-sequenceforcing if potentially $P$-graphic implies forcibly $P$-graphic when $P=\mathcal{F}$-free. We will characterize the degree-sequence-forcing sets $\mathcal{F}$ when $\mathcal{F}$ is of order at most two.

From the above definitions, the following results are immediate.

Proposition 1. In a pair $\{A, B\}$ of graphs, if $A$ is an induced subgraph of $B$, then $\{A, B\}$ is a degree-sequence-forcing set if and only if $\{A\}$ is.

Proposition 2. Given $\mathcal{F}$ any collection of graphs, let $\mathcal{F}^{c}$ denote the collection of graphs which are complements of those in $\mathcal{F}$. Then $\mathcal{F}$ is degree-sequence-forcing if and only if $\mathcal{F}^{c}$ is degree-sequence-forcing.

Let $x, y, u, v$ be four vertices of a graph where $x y, u v$ are edges and $x u, y v$ are not edges. A 2-switch is a graph-theoretic operation which deletes edges $x y, u v$ and adds $x u, y v$. In this article, we shall denote such a 2-switch by $\{x y, u v\} \rightrightarrows\{x u, y v\}$. The diagrams showing such a 2-switch shall have $x y, u v$ as dotted edges before the 2-switch, and $x u, y v$ as double edges after the 2-switch. Note that for a 2-switch, the induced subgraph on $x, y, u, v$ must be $2 K_{2}, C_{4}$, or $P_{4}$. Hence, any $\left\{2 K_{2}, C_{4}, P_{4}\right\}$-free graph admits no 2 -switch. Also, note that any 2 -switch preserves the degree of each vertex of the original graph. The following well-known result will be fundamental in what follows.

Theorem 3 (Fulkerson et al. [8]). Graphs $H$ and $H^{\prime}$ on the same vertex set have $d_{H}(v)=d_{H^{\prime}}(v)$ for every vertex $v$ if and only if $H^{\prime}$ can be obtained by performing a sequence of 2-switches on $H$.

\section{FORBIDDEN PAIRS GENERATING CLASSES OF UNIGRAPHS}

A graph is said to be a unigraph if it is the only realization, up to isomorphism, of its degree sequence. Characterizations of unigraphs and algorithms for testing if a sequence is unigraphic were given by Koren [13], Li [14], and Kleitman and $\mathrm{Li}[12]$.

We make some initial observations. Observation 4 is useful in showing that a set $\mathcal{F}$ is degree-sequence-forcing.

Observation 4. Given any set $\mathcal{F}$ of graphs, if every $\mathcal{F}$-free graph is a unigraph, then $\mathcal{F}$ is degree-sequence-forcing.

The following proposition easily follows from the previous observation. 
Proposition 5. Let $F$ be one of $\left\{K_{1}\right\},\left\{K_{2}\right\}$, or $\left\{2 K_{1}\right\}$. Then every F-free graph is a unigraph. Hence, $\left\{K_{1}\right\},\left\{K_{2}\right\}$, and $\left\{2 K_{1}\right\}$ are degree sequence forcing sets.

We now prove a similar proposition about the pairs $\mathcal{F}$ of graphs where the $\mathcal{F}$-free graphs are unigraphs.

Proposition 6. The following pairs are degree-sequence-forcing:

(i) $\{A, B\}$, where $A$ is one of $K_{1}, K_{2}$, or $2 K_{1}$, and $B$ is arbitrary;

(ii) $\left\{P_{3}, K_{3}\right\},\left\{P_{3}, K_{3}+K_{1}\right\},\left\{P_{3}, K_{3}+K_{2}\right\},\left\{P_{3}, 2 K_{2}\right\},\left\{P_{3}, K_{2}+K_{1}\right\}$;

(iii) $\left\{K_{2}+K_{1}, 3 K_{1}\right\},\left\{K_{2}+K_{1}, K_{1,3}\right\},\left\{K_{2}+K_{1}, K_{2,3}\right\},\left\{K_{2}+K_{1}, C_{4}\right\}$;

(iv) $\left\{K_{3}, 3 K_{1}\right\}$.

\section{Proof.}

(i) This follows easily from Proposition 5 .

(ii) Note that a graph is $P_{3}$-free if and only if it is a disjoint union of complete graphs. A $\left\{P_{3}, K_{3}\right\}$-free graph thus has every component a copy of $K_{2}$ or $K_{1}$ and therefore must be a unigraph. A $\left\{P_{3}, K_{3}+K_{1}\right\}$-free graph falls into one of two cases: in the first case, the graph is $K_{3}$-free, and hence must be a unigraph, as we have seen. In the second case, the graph induces $K_{3}$ and so contains at most one component, is complete and therefore is a unigraph. A $\left\{P_{3}, K_{3}+K_{2}\right\}$-free graph either contains no $K_{3}+K_{1}$ and hence is a unigraph; or the graph does contain an induced $K_{3}+K_{1}$, in which case the component containing the triangle is the only nontrivial component, and hence the graph is a unigraph. A graph which is $\left\{P_{3}, 2 K_{2}\right\}$-free or $\left\{P_{3}, K_{2}+K_{1}\right\}$-free is clearly $\left\{2 K_{2}, C_{4}, P_{4}\right\}$-free, and as such admits no 2switch; such graphs are clearly unigraphs. In each of the cases described, the class of graphs resulting from forbidding the respective pair of induced subgraphs is a subset of the class of unigraphs; by Observation 4, each of these pairs is a degree-sequence-forcing set.

(iii) Each of these pairs contains the complements of a pair of graphs from (ii), so by Proposition 2 we have that these pairs are degree-sequence-forcing.

(iv) By Ramsey's Theorem, each of the $\left\{K_{3}, 3 K_{1}\right\}$-free graphs contains at most 5 vertices. Direct verification shows that each $\left\{K_{3}, 3 K_{1}\right\}$-free graph is a unigraph, so Observation 4 implies that $\left\{K_{3}, 3 K_{1}\right\}$ is a degree-sequenceforcing set.

Each class obtained by forbidding a pair listed above contains graphs with very strict structural requirements, so it is not surprising that these pairs should be degreesequence-forcing. It may be surprising, however, to learn that, other than the pair $\left\{2 K_{2}, C_{4}\right\}$, these are the only degree-sequence-forcing pairs of graphs. We devote the rest of this article to proving this result.

We first state a result found about the $\left\{2 K_{2}, C_{4}\right\}$-free graphs. 
Theorem 7 (Maffray and Preissman [15]). A graph $G$ with degree sequence $d(G)=\left(d_{1}, \ldots, d_{n}\right)$ in nonincreasing order is $\left\{2 K_{2}, C_{4}\right\}$-free if and only if

$$
\sum_{i=1}^{p} d_{i}=p(p-1)+\sum_{i=p+1}^{n} d_{i},
$$

where $p=\max \left\{i: d_{i} \geq i-1\right\}$, or

$$
\sum_{i=1}^{q} d_{i}=q(q+4)+\sum_{i=q+6}^{n} d_{i}
$$

and $d_{q+1}=\cdots=d_{q+5}=q+2$, where $q=\max \{i: i \geq i+4\}$.

From this, we see that $\left\{2 K_{2}, C_{4}\right\}$ is indeed a degree-sequence-forcing set. We now prove that no other pairs are degree-sequence-forcing.

\section{SWITCHING AND THE SIEVE METHOD}

Definition 8. Given a pair $\{A, B\}$ of graphs, we say that $A$ switches to $B$ if whenever $H$ and $H^{\prime}$ are two graphs such that $H$ induces $A, H^{\prime}$ is $A$-free, and $H^{\prime}$ can be obtained by performing a single 2-switch on $H$, we have that $H^{\prime}$ induces $B$.

Proposition 9. Let $A$ and $B$ be any two graphs. The following statements are equivalent:

(i) A switches to $B$ and $B$ switches to $A$.

(ii) If $H$ and $H^{\prime}$ are any two graphs having the same degree sequence such that $H$ induces $A$ and $H^{\prime}$ is $A$-free, then $H^{\prime}$ induces $B$. Likewise, if $H^{\prime}$ induces $B$ and $H$ is $B$-free, then $H$ induces $A$.

(iii) $\{A, B\}$ is a degree-sequence-forcing pair.

\section{Proof.}

(i) $\Longrightarrow$ (ii): Suppose that $H$ and $H^{\prime}$ are two arbitrary graphs with the same degree sequence such that $H$ induces $A$ and $H^{\prime}$ is $A$-free. By Theorem 3, there exists a sequence of $k 2$-switches which, when applied to $H$, results in $H^{\prime}$. For $i=0,1, \ldots, k$, let $H_{i}$ denote the graph obtained after the $i$ th 2-switch, so that $H=H_{0}$ and $H^{\prime}=H_{k}$. Let $j$ be the last index such that $H_{j}$ induces $A$. Then $H_{j+1}$ is $A$-free and since $A$ switches to $B, H_{j+1}$ must induce $B$. If there exists a least index $j^{\prime}>j$ such that $H_{j^{\prime}}$ does not induce $B$, then since $B$ switches to $A, H_{j^{\prime}}$ must induce $A$. This contradicts our choice of $j$ as the last index such that $H_{j}$ induces $A$. Hence $H_{i}$ induces $B$ for all $i \geq j$, and specifically $H^{\prime}=H_{k}$ induces $B$. Similarly, if $H^{\prime}$ induces $B$ and $H$ is $B$-free, then $H$ induces $A$.

(ii) $\Longrightarrow$ (iii): Suppose that $\pi$ is any graphic sequence having a realization $G$ which is $\{A, B\}$-free, and let $G^{\prime}$ be any other realization of $\pi$. If $G^{\prime}$ induces $A$, 
then by (ii) $G$ must induce $B$, which is a contradiction. Similarly, $G^{\prime}$ cannot induce $B$. Hence, $G^{\prime}$ is $\{A, B\}$-free, and since $G^{\prime}$ was arbitrary, $\pi$ is forcibly $\{A, B\}$-free. Since $\pi$ was also arbitrary, $\{A, B\}$ is a degree-sequence-forcing pair.

(iii) $\Longrightarrow$ (i): Suppose that $H$ and $H^{\prime}$ are two graphs such that $H$ induces $A, H^{\prime}$ is $A$-free, and $H^{\prime}$ can be obtained by performing a single 2-switch on $H$. If $H^{\prime}$ is $B$-free, then $d\left(H^{\prime}\right)=d(H)$ is potentially but not forcibly $\{A, B\}$-free. This is a contradiction of the assumption that $\{A, B\}$ is a degree-sequenceforcing pair. Hence, $H^{\prime}$ induces $B$, and since $H$ and $H^{\prime}$ were arbitrary, $A$ switches to $B$. Similarly, $B$ switches to $A$.

As a consequence of the equivalences in Proposition 9, we can use a kind of "sieve method" to find the possible graphs $B$ which can pair with a graph $A$ to form a degree-sequence-forcing set. The method is to find several pairs of graphs $\left(H, H^{\prime}\right)$ such that $d(H)=d\left(H^{\prime}\right), H$ induces $A$, and $H^{\prime}$ is $A$-free. Then for $\{A, B\}$ to form a degree-sequence-forcing set, $B$ must be induced in each of the $H^{\prime}$ s. The induced subgraphs common to all $H^{\prime}$ s give the possible choices for $B$.

Proposition 10. Other than the pairs listed in Proposition 6, there are no degreesequence-forcing pairs in which one graph has 3 or fewer vertices.

Proof. We noted in Proposition 6 that $K_{2}$ or $2 K_{1}$ can be paired with any graph to form a degree-sequence-forcing pair. Suppose first that $\left\{K_{3}, B\right\}$ is a degreesequence-forcing set. The graphs $H_{1}=K_{3}+K_{2}$ and $H_{1}^{\prime}=P_{5}$ are such that $H_{1}$ induces $K_{3}, H_{1}^{\prime}$ is $K_{3}$-free, and $d\left(H_{1}\right)=d\left(H_{1}^{\prime}\right)$. By Proposition 9, $B$ must be induced in $P_{5}$. Similarly, letting $H_{2}$ be the house graph shown in Figure 1 and $H_{2}^{\prime}=K_{2,3}$ demonstrates that $B$ must be induced in $K_{2,3}$. The only induced subgraphs on 3 or more vertices common to both $P_{5}$ and $K_{2,3}$ are $P_{3}$ and $3 K_{1}$; hence, $K_{3}$ belongs to no degree-sequence-forcing pair other than one whose other element is $K_{1}$, $K_{2}, 2 K_{1}, P_{3}$, or $3 K_{1}$. Taking complements, Proposition 2 shows that $\left\{3 K_{1}, B\right\}$ is a degree-sequence-forcing set if and only if $B$ is one of $K_{1}, 2 K_{1}, K_{2}, K_{2}+K_{1}$, or $K_{3}$.

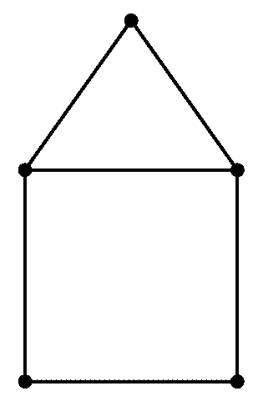

FIGURE 1. The house graph. 

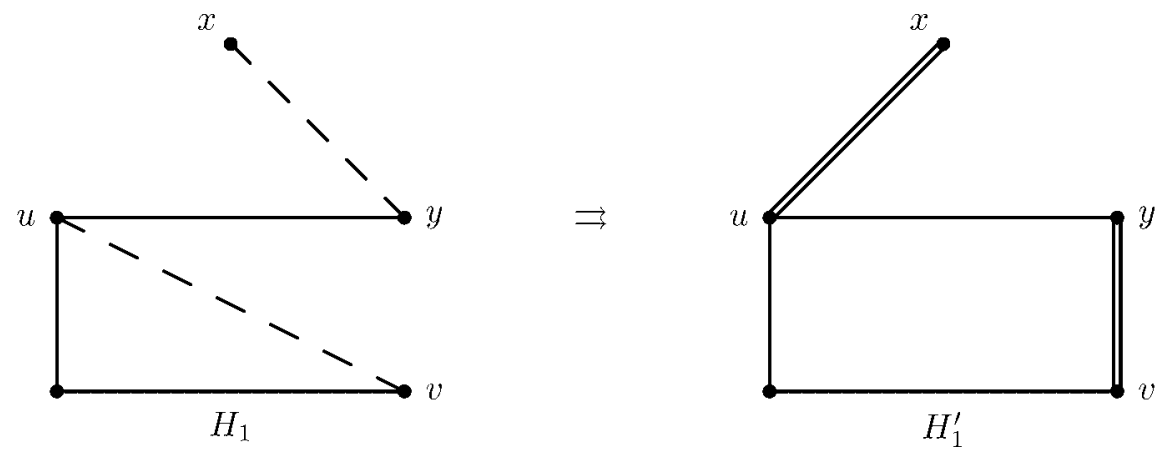

FIGURE 2. The graphs $H_{1}$ and $H_{1}^{\prime}$ from Proposition 11.

Suppose now that $\left\{P_{3}, B\right\}$ is a degree-sequence-forcing pair. If we let $H=P_{5}$ and $H^{\prime}=K_{3}+K_{2}$, then by Proposition $9 B$ must be induced in $K_{3}+K_{2}$, and so must be one of $K_{1}, K_{2}, 2 K_{1}, K_{3}, K_{2}+K_{1}, 2 K_{2}, K_{3}+K_{1}$, or $K_{3}+K_{2}$. Proposition 2 implies that $\left\{K_{2}+K_{1}, B\right\}$ is degree-sequence-forcing only if $B$ is one of $K_{1}, 2 K_{1}$, $K_{2}, 3 K_{1}, P_{3}, C_{4}, K_{3,1}$, or $K_{3,2}$.

We now turn our attention to sets $\{A, B\}$ where both $A$ and $B$ have at least 4 vertices.

Proposition 11. The pair $\left\{2 K_{2}, B\right\}$, where $B$ contains at least 4 vertices, is degreesequence-forcing if and only if $B$ is $C_{4}$; the pair $\left\{C_{4}, B\right\}$, where $B$ contains at least 4 vertices, is degree-sequence-forcing if and only if $B$ is $2 K_{2}$.

Proof. Let $H_{1}$ be the left graph shown in Figure 2, and form the $2 K_{2}$-free graph $H_{1}^{\prime}$ by performing the 2-switch $\{x y, u v\} \rightrightarrows\{x u, v y\}$. Let $H_{2}$ be the left graph shown in Figure 3, and form the $2 \mathrm{~K}_{2}$-free graph $\mathrm{H}_{2}^{\prime}$ by performing the 2-switch $\{x v, u y\} \rightrightarrows\{x y, u v\}$. Let $H_{3}$ be the left graph shown in Figure 4, and form the $2 K_{2}$-free graph $H_{3}^{\prime}$ by performing the 2-switch $\{x v, u y\} \rightrightarrows\{x y, u v\}$. Note that $2 K_{2}$ is induced in each of $\mathrm{H}_{1}, \mathrm{H}_{2}$, and $\mathrm{H}_{3}$. If $\left\{2 \mathrm{~K}_{2}, \mathrm{~B}\right\}$ is degree-sequence-forcing, then $B$ must be induced in each of $H_{1}^{\prime}, H_{2}^{\prime}$, and $H_{3}^{\prime}$. The only induced subgraph with at least 4 vertices common to $H_{1}^{\prime}, H_{2}^{\prime}$, and $H_{3}^{\prime}$ is $C_{4}$, so $B$ must be $C_{4}$.
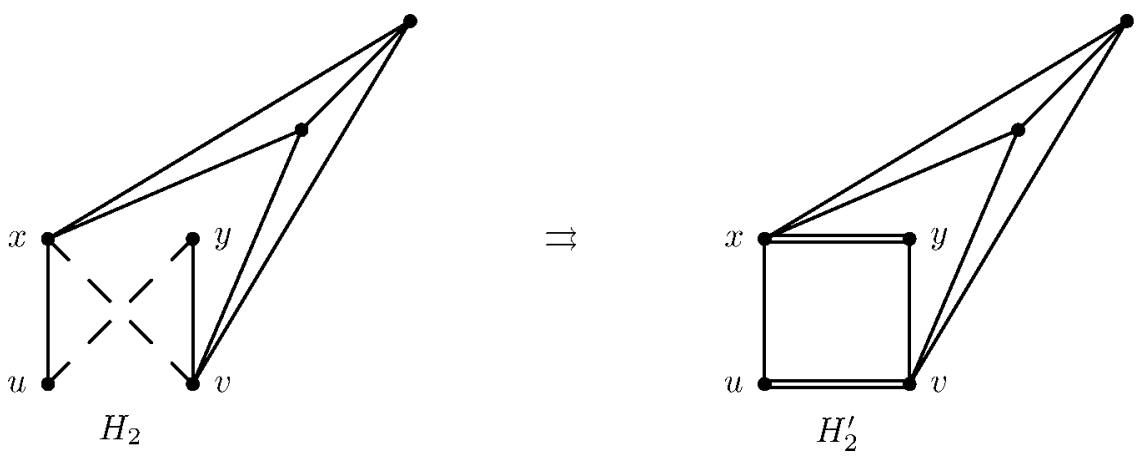

FIGURE 3. The graphs $H_{2}$ and $H_{2}^{\prime}$ from Proposition 11. 


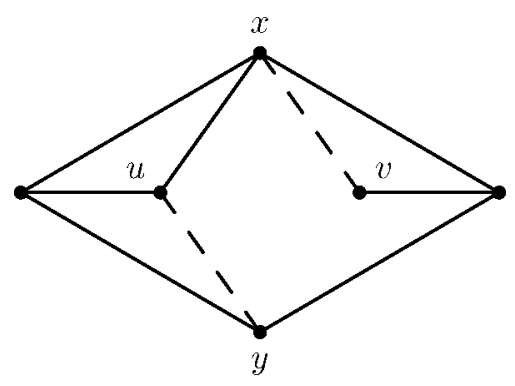

$H_{3}$

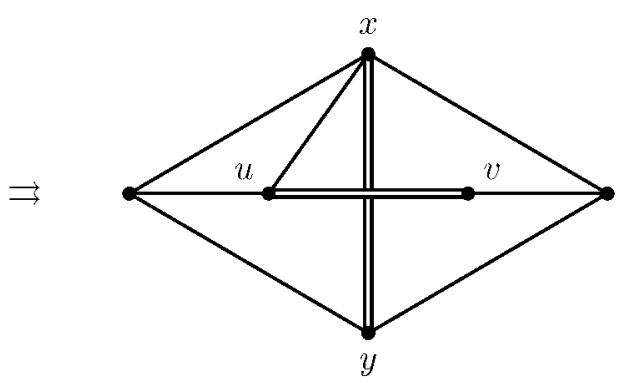

$H_{3}^{\prime}$

FIGURE 4. The graphs $H_{3}$ and $H_{3}^{\prime}$ from Proposition 11.

Conversely, Theorem 7 implies that the $\left\{2 K_{2}, C_{4}\right\}$-free graphs are characterized by their degree sequences, and hence $\left\{2 K_{2}, C_{4}\right\}$ is a degree-sequence-forcing pair.

By observing that the complement of $C_{4}$ is $2 K_{2}$ and by applying Proposition 2 , $\left\{C_{4}, B\right\}$ is degree-sequence-forcing where $B$ has at least 4 vertices if and only if $B$ is $2 K_{2}$.

Lemma 12. For $n \geq 4$, the graph $n K_{1}$ switches to $B$ if and only if $B$ is an induced subgraph of $K_{2}+(n-2) K_{1}$.

Proof. Let $H$ be a graph inducing $n K_{1}$, and let $U$ be a collection of $n$ vertices in $H$ inducing $n K_{1}$. Let $H^{\prime}$ be an $n K_{1}$-free graph obtained by performing a single 2-switch on $H$. Clearly, such a 2-switch must place an edge between two vertices of $U$, and such a 2-switch can involve at most two vertices of $U$. Then in $H^{\prime}$, the subgraph induced on $U$ is isomorphic to $K_{2}+(n-2) K_{1}$, so $n K_{1}$ switches to every induced subgraph of this graph.

We now show that every graph $B$ to which $n K_{1}$ switches is an induced subgraph of $K_{2}+(n-2) K_{1}$. We first define three pairs of graphs.

Define $H_{1}=K_{2, n}$, and let $x, u, y, v$ be any 4-cycle in the graph, as shown in Figure 5. Form the $n K_{1}$-free graph $H_{1}^{\prime}$ by performing the 2-switch $\{x u, y v\} \rightrightarrows$ $\{x y, u v\}$.
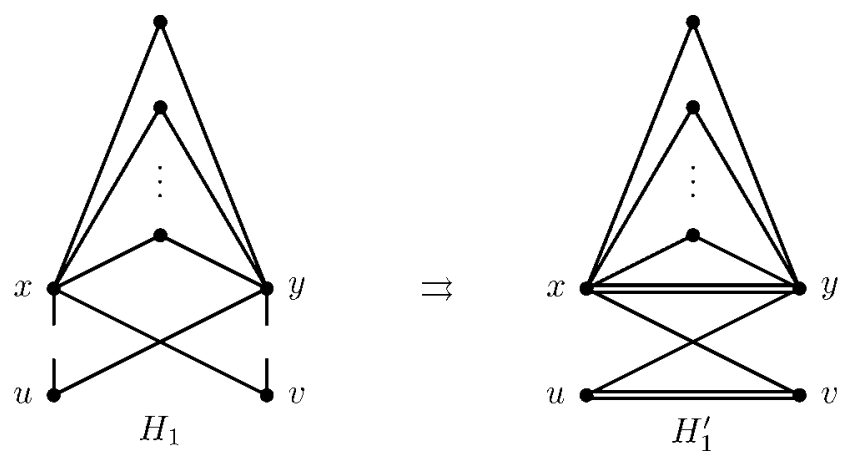

FIGURE 5. The graphs $H_{1}$ and $H_{1}^{\prime}$ from Lemma 12. 


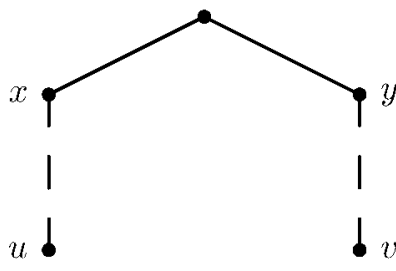

$\mathrm{H}_{2}$
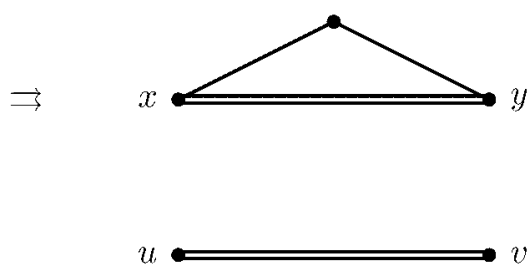

$H_{2}^{\prime}$

FIGURE 6. The graphs $H_{2}$ and $H_{2}^{\prime}$ from Lemma 12.

Let $\mathrm{H}_{2}$ be a collection of $n-3$ isolated vertices and a copy of $P_{5}$, as shown in Figure 6. Form $H_{2}^{\prime}$ by performing the 2-switch $\{x u, y v\} \rightrightarrows\{x y, u v\}$. Note that $H_{2}^{\prime}$ is isomorphic to $K_{3}+K_{2}+(n-3) K_{1}$, and hence has independence number $n-1$.

Finally, let $\mathrm{H}_{3}$ be a collection of $n-4$ isolated vertices and two copies of $P_{3}$, as shown in Figure 7. Form $H_{3}^{\prime}$ by performing the 2-switch $\{x u, y v\} \rightrightarrows\{x y, u v\}$. The graph $H_{3}^{\prime}$ is isomorphic to $P_{4}+K_{2}+(n-4) K_{1}$ and has independence number $n-1$.

Note that $n K_{1}$ is induced in each of $H_{1}, H_{2}$, and $H_{3}$. If $n K_{1}$ switches to a graph $B$, then $B$ must be induced in each of $H_{1}^{\prime}, H_{2}^{\prime}$, and $H_{3}^{\prime}$. We see from $H_{2}^{\prime}$ that $B$ must be a disjoint union of complete graphs, and we see from $H_{3}^{\prime}$ that none of these complete graphs can be of order greater than 2. Furthermore, we see from $H_{1}^{\prime}$ that $B$ cannot contain a copy of $2 K_{2}$, so at most one of the components of $B$ can have more than one vertex. Since $B$ cannot induce $n K_{1}$, there are at most $n-1$ components in $B$, so $B$ is an induced subgraph of $K_{2}+(n-2) K_{1}$, as claimed.

We note that it is clear from the definition that $A$ switches to $B$ if and only if $\bar{A}$ switches to $\bar{B}$, which leads to the following corollary.

Corollary 13. For $n \geq 4$, the graph $K_{n}$ switches to $B$ if and only if $B$ is an induced subgraph of $K_{n}-e$.
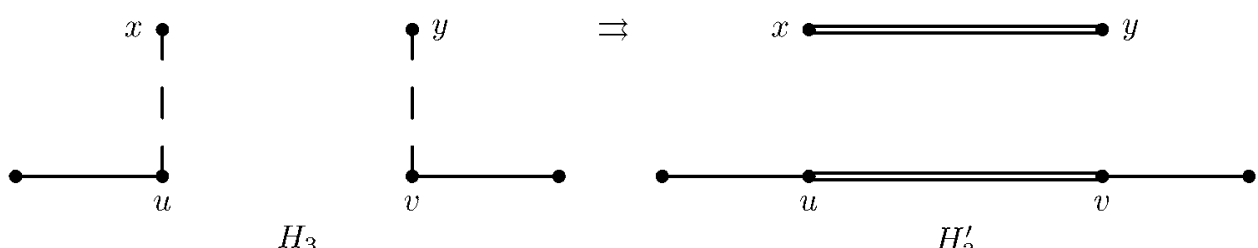

$H_{3}$

$$
H_{3}^{\prime}
$$

FIGURE 7. The graphs $H_{3}$ and $H_{3}^{\prime}$ from Lemma 12 . 


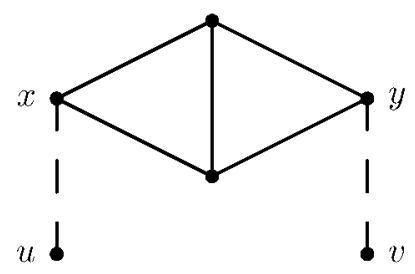

$H_{1}$

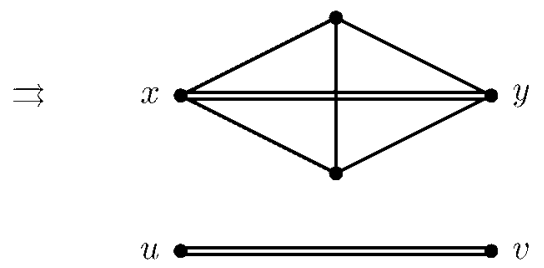

$H_{1}^{\prime}$

FIGURE 8. The graphs $H_{1}$ and $H_{1}^{\prime}$ from Lemma 14.

Lemma 14. For $n \geq 4$, the graph $K_{2}+(n-2) K_{1}$ switches to $B$ if and only if $B$ is $K_{2}$ or $c K_{1}$, where $c \leq n-2$.

Proof. Let $H$ be a graph inducing $K_{2}+(n-2) K_{1}$, and let $H^{\prime}$ be a $K_{2}+(n-$ 2) $K_{1}$-free graph obtained from $H$ after a single 2 -switch. First, note that $K_{2}+(n-$ 2) $K_{1}$ switches to $K_{2}$ since all graphs with an edge switch to $K_{2}$. Furthermore, if $U$ denotes a set of vertices in $H$ inducing $K_{2}+(n-2) K_{1}$, then every 2-switch on $H$ resulting in a $K_{2}+(n-2) K_{1}$-free graph involves exactly two vertices of $U$. Regardless of how many of these two vertices belong to the copy of $K_{2}$, the result after toggling edges in the 2 -switch is that the subgraph induced on $U$ contains an independent set of size at least $n-2$.

We now show that $K_{2}+(n-2) K_{1}$ switches to no other graphs. Let $H_{1}$ denote the graph consisting of $n-4$ isolated vertices and one larger component, as shown in Figure 8 . Form $H_{1}^{\prime}$ by performing the 2-switch $\{x u, y v\} \rightrightarrows\{x y, u v\}$. Note that $H_{1}$ induces $K_{2}+(n-2) K_{1}$, while $H_{1}^{\prime}$ is isomorphic to $K_{4}+K_{2}+(n-4) K_{1}$ and does not induce $K_{2}+(n-2) K_{1}$.

Define $\mathrm{H}_{2}$ as follows: Let $x$ and $y$ be the two vertices in the smaller partite sets in a copy of $K_{1,1, n-2}$. As shown in Figure 9, add two new vertices $u$ and $v$ and the edges the edges $u v, x u$, and $y v$ to form $H_{2}$. Form the graph $H_{2}^{\prime}$ by performing the
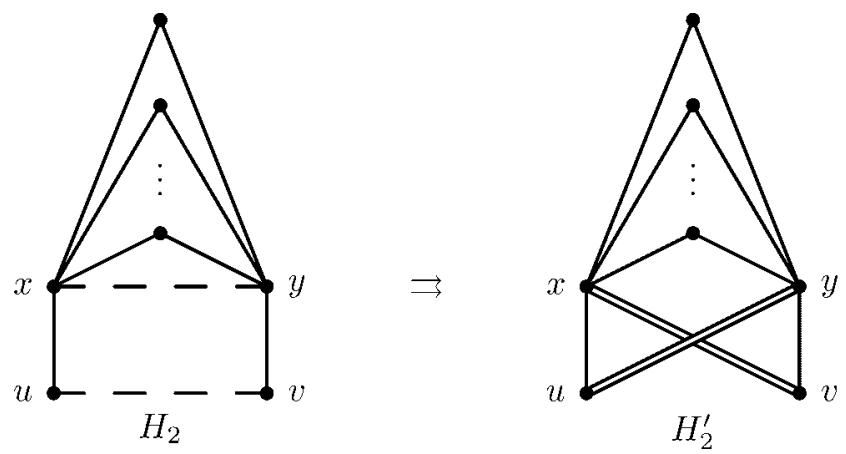

FIGURE 9. The graphs $H_{2}$ and $H_{2}^{\prime}$ from Lemma 14. 
2-switch $\{x y, u v\} \rightrightarrows\{x v, u y\}$. Note that $H_{2}^{\prime}$ is isomorphic to the graph $K_{2, n}$, and, being complete multipartite, is $K_{2}+K_{1}$-free, and hence $K_{2}+(n-2) K_{1}$-free.

Now if $K_{2}+(n-2) K_{1}$ switches to $B, B$ must be an induced subgraph of both $H_{1}^{\prime}$ and $H_{2}^{\prime}$. Since $H_{1}^{\prime}$ is a disjoint union of complete graphs, $B$ must be as well; since $H_{2}^{\prime}$ is a complete bipartite graph, $B$ must also be complete bipartite. The only possibilities are that $B$ is a complete graph of order 2 or less, since $H_{2}^{\prime}$ is triangle-free, or a collection of isolated vertices of order at most $n-2$, since $H_{1}^{\prime}$ has independence number $n-2$.

Corollary 15. For $n \geq 4$, the graph $K_{n}-e$ switches to $B$ if and only if $B$ is $2 K_{1}$ or $K_{c}$, where $c \leq n-2$.

Proposition 16. For $n \geq 4$, the following are not degree-sequence-forcing pairs for any $B$ on 3 or more vertices: $\left\{n K_{1}, B\right\},\left\{K_{n}, B\right\},\left\{K_{2}+(n-2) K_{1}, B\right\}$, and $\left\{K_{n}-e, B\right\}$.

Proof. Suppose that $\left\{n K_{1}, B\right\}$ is a degree-sequence-forcing pair. By Proposition 9 and Lemma $12, B$ is either of the form $m K_{1}$ with $3 \leq m \leq n-1$, or of the form $K_{2}+j K_{1}$ for some $1 \leq j \leq n-2$. If $B=m K_{1}$, then by Lemma 12 we must have $n K_{1}$ a subgraph of a graph of order $m \leq n-1$, a contradiction. Suppose $B=$ $K_{2}+j K_{1}$. The graph $K_{2}+K_{1}$ does not switch to $n K_{1}$ for $n \geq 4$ by Proposition 10 , and by Lemma $14, K_{2}+j K_{1}$ does not switch to $n K_{1}$ for $j \geq 2$ since $n>j$. Thus, by Proposition 9, $\left\{n K_{1}, B\right\}$ is not a degree-sequence-forcing pair for any $B$ on 3 or more vertices. Proposition 2 implies that $\left\{K_{n}, B\right\}$ is likewise not a degreesequence-forcing pair.

Suppose now that the pair $\left\{K_{2}+(n-2) K_{1}, B\right\}$ is degree-sequence-forcing. Lemma 14 immediately gives the result when $n=4$, and when $n>4$, we have that $B$ must be of the form $c K_{1}$, where $3 \leq c \leq n-2$. However, since $n>c, B$ cannot switch to $K_{2}+(n-2) K_{1}$ by Lemma 12 . Thus, for no $B$ on 3 or more vertices is $\left\{K_{2}+(n-2) K_{1}, B\right\}$ degree-sequence-forcing, nor, by Proposition 2, is the pair $\left\{K_{n}-e, B\right\}$.

\section{MONOTONE PARAMETERS}

Another application of the switching idea is to consider the effect of a 2-switch on a given graph parameter. We shall call a graph parameter $p(G)$ monotone if $p(H) \leq p(G)$ whenever $H$ is an induced subgraph of $G$. We now state a fact that shall be useful in the proofs that follow.

Proposition 17. Let $p(G)$ be a monotone parameter and c be a constant. Suppose that whenever $G$ is a graph with $p(G)>c$, there exists a graph $H$ with $G$ as an induced subgraph and a graph $H^{\prime}$ obtained from $H$ by a 2 -switch such that $p\left(H^{\prime}\right)<$ $p(G)$. Then any degree-sequence-forcing set $\mathcal{F}$ has a member $F$ with $p(F) \leq c$.

We illustrate this idea with the following proposition: 
Proposition 18. Any degree-sequence-forcing set $\mathcal{F}$ must contain a forest in which each component is a star.

Proof. Suppose $\mathcal{F}$ contains no forest. Let $F$ have the minimum number of cycles among graphs in $\mathcal{F}$. Let $x y$ be an edge of a cycle in $F$. Form $H$ by adding two new vertices $u, v$ and the edge $u v$. Form $H^{\prime}$ from $H$ by the 2 -switch $\{x y, u v\} \rightrightarrows\{x u, y v\}$. It is clear that $H^{\prime}$ has fewer cycles than $H$ and so is $F$-free and hence $\mathcal{F}$-free. Thus, $\mathcal{F}$ is not degree-sequence-forcing, a contradiction. Hence, $\mathcal{F}$ must contain a forest.

Suppose every forest in $\mathcal{F}$ has a component of diameter at least 3 . Among the forests in $\mathcal{F}$ consider those which minimize the length of a longest path, and among these latter forests choose $F$ having a minimum number of paths of this length. Let $\ell$ denote the length of the longest path in $F$, and let $x y$ be an internal edge of a path in $F$ of length $\ell$. Form $H$ by adding two new vertices $u, v$ and the edge $u v$. Form $H^{\prime}$ from $H$ by the 2 -switch $\{x y, u v\} \rightrightarrows\{x u, y v\}$. It is clear that $H^{\prime}$ is a forest having fewer paths of length $\ell$ than $F$ does, and the longest path in $H^{\prime}$ has length at most $\ell$. It follows that $H^{\prime}$ induces no element of $\mathcal{F}$. Since $H$ induces $F$, we have that $\mathcal{F}$ is not degree-sequence-forcing, a contradiction. Thus, $\mathcal{F}$ must contain a forest in which each component is a star.

The first paragraph of the proof above illustrates the idea of Proposition 17 with $p(G)$ being the number of cycles in $G$ and $c=0$. Note that the statement of Proposition 17 also holds when $p(G)$ takes values in any linearly ordered set, and such a formulation could be used to provide an alternate version of the second paragraph of the proof of Proposition 18.

The following corollary is a simple consequence of Propositions 2 and 18.

Corollary 19. Any degree-sequence-forcing set $\mathcal{F}$ must contain a graph which is the complement of a forest of stars.

Proposition 20. Any degree-sequence-forcing set $\mathcal{F}$ must contain a graph which is a disjoint union of complete graphs.

Proof. Let $p(G)$ denote the number of edges needed to make every component of $G$ complete. Note that $p(G)$ is a monotone parameter. Let $G$ be an arbitrary graph such that $p(G) \geq 1$, and let $x$ and $y$ be two non-adjacent vertices in a component of $G$. Form $H$ by adding to $G$ two new vertices $u, v$ and edges $x u$ and $y v$. Form $H^{\prime}$ from $H$ by the 2 -switch $\{x u, y v\} \rightrightarrows\{x y, u v\}$. It is clear that $p\left(H^{\prime}\right)<p(G)$. By Proposition 17, if $\mathcal{F}$ is degree-sequence-forcing then $\mathcal{F}$ must contain an element $F$ such that $p(F)=0$, that is, a disjoint union of complete graphs.

Corollary 21. Any degree-sequence-forcing set $\mathcal{F}$ must contain a complete multipartite graph. 
Hence, we consider the following families of graphs:

$$
\begin{aligned}
& \mathbb{K}:=\{\text { Union of complete graphs }\} \\
& \mathbb{K}^{c}:=\{\text { Complete multipartite graphs }\} \\
& \mathbb{S}:=\{\text { Forest of stars }\} \\
& \mathbb{S}^{c}:=\{\text { Complements of forests of stars }\}
\end{aligned}
$$

We now characterize all singleton degree-sequence-forcing sets.

Theorem 22. The only degree-sequence-forcing singleton sets $\mathcal{F}$ are $\left\{K_{1}\right\},\left\{K_{2}\right\}$, and $\left\{2 K_{1}\right\}$.

Proof. Let $\mathcal{F}=\{F\}$. Then by Propositions 18 and 20 and Corollaries 19 and 21, $F \in \mathbb{K} \cap \mathbb{K}^{c} \cap \mathbb{S} \cap \mathbb{S}^{c}=\left\{K_{1}, K_{2}, 2 K_{1}\right\}$. Proposition 5 then gives the result.

In characterizing the degree-sequence-forcing pairs, we note that by Propositions 10 and 16 it will be sufficient to characterize the degree-sequence-forcing pairs $\mathcal{F}$ $=\left\{F_{1}, F_{2}\right\}$, where each $F_{i}$ has order at least 4 and is not $K_{n}, K_{n}-e, n K_{1}$, or $K_{2}+(n-2) K_{1}$.

Proposition 23. The only degree-sequence-forcing pair $\mathcal{F}=\left\{F_{1}, F_{2}\right\}$ where the order of each $F_{i}$ is at least 4 is $\left\{2 K_{2}, C_{4}\right\}$.

Proof. We consider which elements of $\mathcal{F}$ are in the families $\mathbb{K}, \mathbb{K}^{c}, \mathbb{S}, \mathbb{S}^{c}$.

Case 1. If $F_{i}$ belongs to all of the above families, that is, $F_{i} \in \mathbb{K} \cap \mathbb{K}^{c} \cap \mathbb{S} \cap \mathbb{S}^{c}$ for $i=1$ or 2 , then $F_{i} \in\left\{K_{1}, K_{2}, 2 K_{1}\right\}$. Hence, $F_{i}$ has order at most 2, which is a contradiction of the hypothesis.

Case 2. If $F_{i}$ belongs to any three of the families, then $F_{i}$ belongs to

$$
\begin{aligned}
\mathbb{K} \cap \mathbb{K}^{c} \cap \mathbb{S} & =\left\{K_{2}, n K_{1}\right\} \\
\text { or } \quad \mathbb{K} \cap \mathbb{K}^{c} \cap \mathbb{S}^{c} & =\left\{2 K_{1}, K_{n}\right\} \\
\text { or } \quad \mathbb{K} \cap \mathbb{S} \cap \mathbb{S}^{c} & =\left\{K_{1}, K_{2}, 2 K_{1}, K_{2}+K_{1}\right\} \\
\text { or } \quad \mathbb{K}^{c} \cap \mathbb{S} \cap \mathbb{S}^{c} & =\left\{K_{1}, K_{2}, 2 K_{1}, P_{3}\right\} .
\end{aligned}
$$

Hence, $F_{i}$ has order at most 3 , contradicting the hypothesis, or is a complete or edgeless graph, contradicting Proposition 16.

Case 3. If $F_{1}$ belongs to exactly two of the families, then $F_{2}$ belongs to the other two families; hence, we may assume

$$
\begin{array}{rlrl}
F_{1} & \in \mathbb{K} \bigcap \mathbb{K}^{c}=\left\{K_{n}, n K_{1}\right\} & \text { and } F_{2} \in \mathbb{S} \bigcap \mathbb{S}^{c}=\left\{K_{1}, K_{2}, 2 K_{1}, K_{2}+K_{1}, P_{3}\right\} \\
\text { or } F_{1} \in \mathbb{K} \bigcap \mathbb{S}^{c}=\left\{K_{a}+K_{1}, 2 K_{1}, K_{n}\right\} & \text { and } F_{2} \in \mathbb{K}^{c} \bigcap \mathbb{S}=\left\{K_{1, b}, K_{2}, n K_{1}\right\} \\
\text { or } F_{1} & \in \mathbb{K} \bigcap \mathbb{S}=\left\{a K_{2}+b K_{1}\right\} & & \text { and } F_{2} \in \mathbb{K}^{c} \bigcap \mathbb{S}^{c}=\left\{K_{2, \ldots, 2,1, \ldots, 1}\right\} .
\end{array}
$$


There are only two cases which do not contradict our assumption on the order of $F_{i}$ or Proposition 16.

Subcase 1. $F_{1}=K_{a}+K_{1}$ and $F_{2}=K_{1, b}$, where $a \geq 3, b \geq 3$. Let $v$ be the isolated vertex in $F_{1}$ and $x y$ be any edge of the clique $K_{a}$. Form $H$ by adding a vertex $u$ and adding the edge $u v$. Form $H^{\prime}$ from $H$ by the 2-switch $\{x y, u v\} \rightrightarrows\{x u, y v\}$. We note that $H^{\prime}$ is both $K_{a}+K_{1}$ and $K_{1, b}$ free. Hence $\mathcal{F}=\left\{F_{1}, F_{2}\right\}$ is not degreesequence-forcing, a contradiction.

Subcase 2. $F_{1}=a K_{2}+b K_{1}$ where $a \geq 2$ and $F_{2}=K_{2, \ldots, 2,1, \ldots, 1}$, with at least two partite sets of size 2 . Let $x y$ and $v z$ be any two edges of $F_{1}$. Form $H$ by adding a vertex $u$ and adding the edges $u x, u v$, and $u z$. Form $H^{\prime}$ from $H$ by the 2 -switch $\{x y, u v\} \rightrightarrows\{x v, y u\} . H^{\prime}$ is $F_{1}$-free and the only subgraph of the form $K_{2, \ldots, 2,1, \ldots, 1}$ with at least two partite sets of size 2 that is induced in $H^{\prime}$ is $C_{4}$. Thus $F_{2}=C_{4}$, and by Proposition 11 we have $F_{1}=2 K_{2}$.

Hence, the result.

Theorem 24. The list of all degree-sequence-forcing pairs is as follows:

(i) $\{A, B\}$, where $A$ is one of $K_{1}, K_{2}$, or $2 K_{1}$, and $B$ is arbitrary;

(ii) $\left\{P_{3}, K_{3}\right\},\left\{P_{3}, K_{3}+K_{1}\right\},\left\{P_{3}, K_{3}+K_{2}\right\},\left\{P_{3}, 2 K_{2}\right\},\left\{P_{3}, K_{2}+K_{1}\right\}$;

(iii) $\left\{K_{2}+K_{1}, 3 K_{1}\right\},\left\{K_{2}+K_{1}, K_{1,3}\right\},\left\{K_{2}+K_{1}, K_{2,3}\right\},\left\{K_{2}+K_{1}, C_{4}\right\}$;

(iv) $\left\{K_{3}, 3 K_{1}\right\}$;

(v) $\left\{2 K_{2}, C_{4}\right\}$.

Proof. Proposition 6 shows that each of the pairs in (i)-(iv) are degreesequence-forcing and by Proposition 10, there are no other degree-sequence-forcing pairs where one graph has 3 or fewer vertices. Proposition 11 shows that $\left\{2 K_{2}, C_{4}\right\}$ is degree-sequence-forcing, and Proposition 23 shows that besides the pair $\left\{2 K_{2}, C_{4}\right\}$, there are no degree-sequence-forcing pairs where each graph contains at least 4 vertices.

\section{FUTURE WORK}

We note that not every superset of a degree-sequence-forcing set is degree-sequenceforcing; consider, for example, the set $\left\{2 K_{2}, C_{4}, K_{1,4}\right\}$ and the three realizations of the degree sequence $(5,4,3,2,2,1,1)$. However, it follows from Proposition 1 that if $\mathcal{F}$ is a family such that the $\mathcal{F}$-free graphs are unigraphs, then $\mathcal{F}$ is a degree-sequence-forcing set, and the union of $\mathcal{F}$ with any collection of graphs is a again degree-sequence-forcing. Other degree-sequence-forcing sets are known to be subsets of larger degree-sequence-forcing sets as well. We thus define a degreesequence-forcing set $\mathcal{F}$ of graphs to be minimal if no proper subset of $\mathcal{F}$ is a degree-sequence-forcing set. It is easy to see there are infinitely many minimal degree-sequence-forcing sets; the set $\mathcal{G}_{n}$ of all graphs on $n$ vertices is clearly degreesequence-forcing, and each $\mathcal{G}_{n}$ contains a minimal degree-sequence-forcing set. 
However, by Theorems 22 and 24, the number of minimal singletons and pairs is finite. This provides motivation for the following theorem, whose proof will appear in [1].

Theorem 25. Given $k \geq 2$, there are only finitely many minimal degree-sequenceforcing $k$-sets.

The authors are currently working on characterizing the degree-sequence-forcing triples. We have completed the characterization of nonminimal degree-sequenceforcing-triples, the only interesting case being where $\left\{2 K_{2}, C_{4}\right\}$ is a subset. We have found nontrivial nonminimal degree-sequence-forcing triples that have not already appeared in the literature. These results will also appear in [1].

Finally, we note that our results in this article and in [1] guarantee the existence of degree sequence characterizations for certain graph classes but never explicitly give such characterizations. Because of the structural simplicity of the graphs in classes forbidding a degree-sequence-forcing singleton or pair, it is a trivial matter to generate a degree sequence characterization for these classes, except in the case of the $\left\{2 K_{2}, C_{4}\right\}$-free graphs; the characterization for this class was found in [15]. Rao describes a method in [19] for finding a degree sequence characterization for the forcibly $P$-free graphs, where $P$ is any hereditary property. This method can therefore generate the degree sequence characterizations we desire, but the characterizations produced contain very large numbers of inequalities. It appears that many of the inequalities may be combined or omitted to produce simple characterizations similar to the one in Theorem 7. It would be interesting to also generate simple degree sequence characterizations for classes forbidding larger degree-sequence-forcing sets.

\section{ACKNOWLEDGMENTS}

We thank the anonymous referees for their helpful comments. The research work of M.D.B. was partially supported by the National Science Foundation VIGRE grant DMS 9983160.

\section{REFERENCES}

[1] M. D. Barrus, S. G. Hartke, and M. Kumbhat, Nonminimal degree-sequenceforcing triples (preprint).

[2] M. Chudnovsky, N. Robertson, P. D. Seymour, and R. Thomas, The strong perfect graph theorem, Ann Math (2), 164(1) (2006), 51-229.

[3] A. A. Chernyak, Zh. A. Chernyak, and R. I. Tyshkevich, On forcibly hereditary $P$-graphical sequences, Discrete Math 64(2-3) (1987), 111-128.

[4] V. Chvátal and P. L. Hammer, Aggregation of inequalities in integer programming, In: Studies in Integer Programming, P. L. Hammer, E. L. 
Johnson, B. H. Korte, and G. L. Nemhauser (Editors), North-Holland, New York, 1977, pp. 145-162.

[5] R. J. Faudree, R. J. Gould, M. S. Jacobson, and L. L. Lesniak, Characterizing forbidden clawless triples implying Hamiltonian graphs, Discrete Math 249(13) (2002), 71-81.

[6] R. J. Faudree, R. J. Gould, and M. S. Jacobson, Forbidden triples implying Hamiltonicity: for all graphs, Discuss Math Graph Theory 24(1) (2004), 4754.

[7] S. Földes and P. L. Hammer, On split graphs and some related questions, Problèmes Combinatoires et Théorie Des Graphes, 138-140, Orsay, France, 1976, Colloques internationaux C.N.R.S. 260.

[8] D. R. Fulkerson, A. J. Hoffman, and M. H. McAndrew, Some properties of graphs with multiple edges, Canad J Math 17 (1965), 166-177.

[9] R. J. Gould, T. Łuczak, and F. Pfender, Pancyclity of 3-connected graphs: Pairs of forbidden subgraphs, J Graph Theory 47(3) (2004), 183-202.

[10] P. L. Hammer, T. Ibaraki, and B. Simeone, Degree sequences of threshold graphs, In: Proceedings of the Ninth Southeastern Conference on Combinatorics, Graph Theory, and Computing (Florida Atlantic University, Boca Raton, FL, 1978) Congress Numer, XXI, Utilitas Math, Winnipeg, Man, pp. 329-355, 1978.

[11] P. L. Hammer and B. Simeone, The splittance of a graph, Combinatorica 1 (1981), 275-284.

[12] D. J. Kleitman and S.-Y. Li, A note on unigraphic sequences, Studies Appl Math 54(4) (1975), 283-287.

[13] M. Koren, Sequences with a unique realization by simple graphs, J Combin Theory B 21(3) (1976), 235-244.

[14] S.-Y. Li, Graphic sequences with unique realization, J Combin Theory B 19(1) (1975), 42-68.

[15] F. Maffray and M. Preissmann, Linear recognition of pseudo-split graphs, Discrete Appl Math 52 (1994), 307-312.

[16] T. A. McKee, Forbidden subgraphs in terms of forbidden quantifiers, Notre Dame J Formal Logic 19 (1978), 186-188.

[17] B. Randerath, 3-colorability and forbidden subgraphs, I: Characterizing pairs, Discrete Math 276(1-3) (2004), 313-325.

[18] S. B. Rao, A survey of the theory of potentially $P$-graphic and forcibly $P$ graphic degree sequences, Combinatorics and Graph Theory: Proceedings of the Symposium Held at the Indian Statistical Institute, Calcutta, February 25-29. Springer-Verlag, Lecture Notes in Mathematics, Vol. 885, 1980, pp. 417-440.

[19] S. B. Rao, Towards a theory of forcibly hereditary $P$-graphic sequences, Combinatorics and Graph Theory: Proceedings of the Symposium Held at the 
Indian Statistical Institute, Calcutta, February 25-29. Springer-Verlag, Lecture Notes in Mathematics, Vol. 885, 1980, pp. 441-458.

[20] R. I. Tyshkevic, A. A. Chernyak, and Zh. A. Chernyak, Forcibly p-graphic sequences, Dokl Akad Nauk BSSR 29(8) (1985), 677-680, 763 (in Russian).

[21] D. B. West, Introduction to Graph Theory, Second Edition, Prentice Hall, Inc., Upper Saddle River, NJ, 2000.

[22] I. E. Zverovich, Proof of a theorem of Rao on forcibly planar degree sequences, Vestsi Akad Navuk BSSSR Ser Fiz-Mat Navuk 4 (1987), 113 (in Russian).

[23] I. E. Zverovich, Forcibly 3-colorable degree sequences, Vychisl Sistemy, Voprosy Algoritm Anal Strukturn Inform 119(34-42) (2004), 113-114.

[24] I. E. Zverovich and O. P. Urbanovich, 2-matroid graphs and power sequences, Vestsi Akad Navuk BSSR Ser Fiz-Mat Navuk (3) (1990), 35-39, 124. 